Physics Vol. 2, No. 6, pp. 273-289, 1966. Physics Publishing Co. Printed in Great Britain.

\title{
THE MAXIMAL CHIRAL GROUP WITH THE QUARK MODEL*
}

\author{
S. OKUBO ${ }^{\dagger}$, R.E. MARSHAK and H. GOLDBERG \\ University of Rochester, Rochester, N.Y. \\ and \\ C. RYAN \\ Institute for Advanced Study, Dublin \\ (Received 9 Feburary 1966)
}

\section{Introduction}

IN a series of papers [1-3], the first two authors attempted to use the quark model as a probe of higher symmetries for the hadrons (and hopefully for the leptons as well). It is immediately evident that a triplet of quark fields with common mass $m$ yields an internal $S_{3}$ group structure (in addition to invariance under the Poincaré group $\mathscr{P}$ and the discrete transformations $P, C$, $T$ ); that is to say, the general quark Lagrangian $\mathscr{L}$ :

$$
\begin{aligned}
& \mathscr{L}=\mathscr{L}_{k}+\mathscr{L}_{m}+\mathscr{L}_{I} \\
& \mathscr{L}_{k}=\sum_{i=1}^{3} \bar{\psi}_{i} \gamma_{\mu} \partial_{\mu} \Psi_{i} \\
& \mathscr{L}_{m}={ }_{m} \sum_{i=1}^{3} \bar{\psi}_{i} \Psi_{i} \\
& \mathscr{L}_{I}=\sum_{n=1}^{5} g_{n}\left(\sum_{i=1}^{3} \bar{\Psi}_{i} Q_{n} \Psi_{i}\right)^{2}
\end{aligned}
$$

where $Q_{n}=$ scalar $(S)$, pseudoscalar $(P)$, vector $(V)$, axial vector $(A)$, or tensor $(T)$, is invariant under $\mathscr{P} \otimes \mathscr{D} \otimes S U_{3}$ [4]. However, it was shown in the very first paper that $\mathscr{L}$ is invariant under a higher (chiral) symmetry group if one chooses $m=0$ in $\mathscr{L}_{m}$ and selects a particular $Q_{n}$ in $\mathscr{L}_{I}$. Indeed, the choice $Q_{n}=S, P$ or $T$ led to higher symmetry groups which were physically uninteresting since all their irreducible representations involved $S U_{3}$ representations with both zero and non-zero trialities. Only a combination of $V$ and $A$ four-fermion interactions led to a higher chiral symmetry group which contained irreducible representations involving only $\mathrm{SU}_{3}$ representations with zero triality (which are the only types of representations observed for hadrons). The resulting chiral group, $\mathscr{P} \otimes \bar{W}_{3}$ (where $\bar{W}_{3} \equiv U_{3}^{(+)} \otimes U_{3}^{(-)}$with $U_{3}^{(+)}\left(U_{3}^{(-)}\right)$spanned by the three positive (negative) chiral projected fields: $\left.\left(1 \pm \gamma_{5}\right) \psi_{i}\right)$ mixes

* This work was supported in part by the U.S. Atomic Energy Commission.

$\dagger$ John Simon Guggenheim Fellow. 
parity with $\mathrm{SU}_{3}$ and can be used to deduce mass relations [5] between hadrons of opposite parity (and the same spin).

Independently of our work, Gell-Mann [6] was led to the $S \bar{W}_{3}$ group [7] by looking for the group generated, under equal-time commutation, by the space integrals of the fourth components of the vector and axial vector weak hadron currents. It is simple to show that the space integrals of the fourth components of the vector and axial vector currents defined in terms of quark fields lead to the equal-time commutation relations postulated by Gell-Mann. One might inquire into the relative advantages of the two formulations. It seems that the quark model is generally more useful if one is interested in the higher symmetry group as a "spectrum-generating" group [8] since it lends itself naturally to a prescription for the tensor structure (T.S.) of the symmetry-breaking interactions which then yields the desired mass relations among the hadrons. On the other hand, current algebras are more useful as "transition operator" groups [8], in which role they may be employed to perform dynamical calculations - which at the present stage is really not possible with the quark model.

The usefulness of the quark model as a probe of higher symmetry groups which belong to the "spectrum-generating" class was demonstrated anew by our study of the consequences of a nonchiral rather than a chiral decomposition of the triplet of quark fields. We showed [2] that with non-chiral decomposition one may obtain a compact group [9] as large as $W_{6}=U_{6}^{(+)} \otimes U_{6}^{(-)}$ [where the plus and minus signs now refer to the non-chiral projected fields: $\left(1 \pm \gamma_{4}\right) \psi_{i}$ ]. This non-chiral $W_{6}$ group, which we first derived on the basis of the quark model, has recently become quite popular [10], and contains the collinear and coplanar groups as sub-groups [11]. In contrast, the generalization of the current algebra approach [12] led to the chiral $W_{6}$ group which has not been particularly useful as a "spectrum-generating" group. It is to be noted that in the quark model, $\mathscr{L}_{m}$ and $\mathscr{L}_{I}$ (with $g_{n}=S$ or $P$ ) are invariant under the non-chiral $W_{6}$ group.

While the non-chiral $W_{6}$ group is very attractive as a "spectrum-generating" group, it suffers from the usual difficulties of relativistic $S U_{6}$ theories (which mix the Poincare and $S U_{3}$ internal symmetry groups) - the conflict between unitarity and crossing [13], the need to work with an infinite dimensional Lie algebra [2] (if one wishes to save Lorentz covariance), and so on. Moreover, the current algebra which corresponds to the non-chiral $W_{6}$ group involves currents (tensor, scalar, etc.) which do not play a direct physical role in either the electromagnetic or weak interactions. This has led to the point of view [8] that the non-chiral $W_{6}$ group is the maximal compact "spectrum-generating" group whereas the chiral $\bar{W}_{3}$ group is the maximal compact "transition operator" group for the hadrons. From the point of view of the quark model, this would imply that somehow the mass term is more important than the kinetic part of the Lagrangian (1) when one deals with the mass spectra of the hadrons and the reverse is true [14] when one is concerned with the algebra of the physical ( $V$ and $A$ ) currents. While this is a possible attitude to take, it would seem more satisfactory if the quark model could suggest a higher symmetry group which would serve the purpose of generating both the mass spectra of the hadrons as well as a physically interesting algebra of currents. In Section 2, we carry forth this program and determine the maximal chiral group suggested by the quark model which mixes spin and unitary spin in the mass spectra of hadrons, while in Section 3 this group is discussed as a current algebra. Finally, the Appendix contains a rather complete tabulation of the various higher symmetry groups obtainable with the quark model and justifies our statement concerning the maximal chiral and non-chiral groups which are possible.

\section{2. $\tilde{W}_{3} \otimes N_{3}$ Group as a Spectrum-Generating Group}

Our starting point is the Lagrangian (1) with $m=0$, rewritten in the form: 


$$
\begin{aligned}
& \mathscr{L}=\mathscr{L}_{K}+\mathscr{L}_{I} \\
& \mathscr{L}_{K}=\sum_{i=1}^{3} \bar{\psi}_{i} \gamma_{\mu} \partial_{\mu} \Psi_{i} \\
& \mathscr{L}_{I}=g_{V}\left(\sum_{i=1}^{3} \bar{\psi}_{i} \gamma_{\mu} \psi_{i}\right)^{2}+g_{A}\left(\sum_{i=1}^{3} \bar{\psi}_{i} \gamma_{\mu} \gamma_{5} \psi_{i}\right)^{2}
\end{aligned}
$$

with $g_{V}, g_{A}$ the vector and axial vector coupling constants. Making the usual chiral decomposition: $\psi_{i}{ }^{(t)}=\frac{\left(i \pm \gamma_{5}\right)}{2} \psi_{i}$, equation (2) is transformed into:

$$
\begin{aligned}
\mathscr{L}_{K} & =\sum_{i=1}^{3}\left\{\bar{\psi}_{i}^{(+)} \gamma_{\mu} \partial_{\mu} \Psi_{i}^{(+)}+\bar{\psi}_{i}^{(-)} \gamma_{\mu} \partial_{\mu} \Psi_{i}^{(-)}\right\} \\
\mathscr{L}_{I} & =g_{V}\left\{\sum_{i=1}^{3} \bar{\psi}_{i}^{-(+)} \gamma_{\mu} \Psi_{i}^{(+)}-\bar{\psi}_{i}^{(-)} \gamma_{\mu} \Psi_{i}^{(-)}\right\}^{2} \\
& +g_{A}\left\{\sum_{i=1}^{3} \Psi_{i}^{(+)} \gamma_{\mu} \Psi_{i}^{(+)}+\bar{\psi}_{i}^{(-)} \gamma_{\mu} \Psi_{i}^{(-)}\right\}^{2}
\end{aligned}
$$

If $g V \neq g_{A}$, equation (3) is invariant under the group $\bar{W}_{3}=U_{3}^{(+)} \otimes U_{3}^{(-)}$where the two $U_{3}$ groups, as remarked before, refer respectively to the positive $\left(\psi_{i}^{(+)}\right)$and negative $\left(\psi_{i}^{(-)}\right.$chiral projections of the triplet of quark fields. There is no mixing with the Poincaré group, $\mathscr{P}$. so that we are in fact dealing with the group $\bar{W}_{3} \otimes \mathscr{P} ;$ this was the case previously considered in $I$.

Now suppose we choose $g_{V}=g_{A}=g$, a choice made attractive by the invariance of the resulting $(V-A)$ interaction under Fierz rearrangement [15]; then equation (3) assumes the compact form:

$$
\begin{aligned}
& \mathscr{L}=\mathscr{L}\left[\Psi_{i}^{(+)}\right]+\mathscr{L}\left[\Psi_{i}^{(-)}\right] \\
& \mathscr{L}\left[\Psi_{i}^{(+)}\right]=\sum_{i=1}^{3}\left\{\bar{\psi}_{i}^{(+)} \gamma_{\mu} \partial_{\mu} \Psi_{i}^{(+)}\right\}+g\left[\sum_{i=1}^{3} \Psi_{i}^{-(+)} \gamma_{\mu} \Psi_{i}^{(+)}\right]^{2} \\
& \mathscr{L}\left[\psi_{i}^{(-)}\right]=\sum_{i=1}^{3}\left\{\bar{\psi}_{i}^{(-)} \gamma_{\mu} \partial_{\mu} \Psi_{i}^{(-)}\right\}+g\left[\sum_{i=1}^{3} \psi_{i}^{-(-)} \gamma_{\mu} \Psi_{i}^{(-)}\right]^{2}
\end{aligned}
$$

We observe that equation (4) separates completely into two distinct parts, one part depending only on $\psi_{i}^{(+)}$and the other part depending only on $\psi_{i}^{(-)}$. It follows that the equations of motion for $\psi_{i}^{(+)}$and $\psi_{i}^{(-)}$are completely decoupled and hence the Lagrangian (4) when integrated over 
space-time is invariant under two independent Poincaré transformations $\mathscr{P}^{(+)}$and $\mathscr{P}^{(-)}$operating on $\psi_{i}^{(+)}(x)$ and $\psi_{i}^{(-)}(x)$ respectively, in addition to its invariance under the two independent $U_{3}^{(+)}$and $U_{3}^{(-)}$transformations. If we write $[16] \mathscr{B}=\mathscr{P}^{(+)} \otimes \mathscr{P}^{(-)}$, then we can say that the $(V-A)$ theory (defined by equation 4$)$ is invariant under the larger group $\bar{W}_{3} \otimes \mathscr{B}$ in contrast to the general $(V, A)$ case (defined by equation 3 ) which is invariant under $\bar{W}_{3} \otimes \mathscr{P}$.

We now propose to consider the mixed chiral group $\bar{W}_{3} \bigotimes \mathscr{B}$ as a "spectrum-generating" group and to remove the mass degeneracy associated with each irreducible representation of this large group by switching on the (common) mass term in the Lagrangian, namely:

$$
\mathscr{L}_{m}=m \sum_{i=1}^{3} \bar{\psi}_{i} \psi_{i}=m \sum_{i=1}^{3}\left[\bar{\psi}_{i}^{(+)} \psi_{i}^{(-)}+\bar{\psi}_{i}^{(-)} \psi_{i}^{(+)}\right]
$$

The symmetry-breaking $\mathscr{L}_{m}$ collapses the two Poincaré spaces $\mathscr{P}^{(+)}$and $\mathscr{P}^{(-)}$into a single Poincare space and the two unitary spaces $U_{3}^{(+)}$and $U_{3}^{(-)}$into a single $U_{3}$ so that the sub-group of invariance [17] is $U_{3} \otimes \mathscr{P}$. In point of fact, the symmetry-breaking mass term performs another very useful function; as Coleman [18] has pointed out, the invariance of our Lagrangian (4) under the product of the two Poincaré groups $\mathscr{P}^{(+)}$and $\mathscr{P}^{(-)}$implies the following relation between the four-momentum $P$ of a hadron and the four-momenta $p_{+}$and $p_{-}$respectively of the positive and negative chiral quark fields:

$$
p^{2}=\left(p_{+}+p_{-}\right)^{2}
$$

The above relation will lead to a continuous range of mass values unless $p_{+}$or $p_{-}$is identically zero. Since we know that the free Lagrangian $\left(\mathscr{L}_{K}+\mathscr{L}_{m}\right)$ does not yield a mass continuum, we believe that the symmetry-breaking mass term (5) must somehow help us out of this difficulty although its precise mechanism is not clear. Coleman [19] had already pointed out a related difficulty for any group (containing $\mathrm{SU}_{3} \otimes \mathscr{P}$ ) which mixes space-time with internal symmetries, to wit one is led to a continuum of masses or to a continuously infinite number of irreducible representations of $S U_{3} \otimes \mathscr{P}$ associated with the same mass $m$. Despite this objection, the nonrelativistic $S_{6}$ group has been subjected to symmetry-breaking terms which formally lead to relations among discrete masses. We take a similarly optimistic attitude and remark, moreover, that our quark model with chiral decomposition may be used as a guiding principle to suggest the larger group $\bar{W}_{3} \otimes \mathscr{B}$ and that this enlarged group could equally well be indentified as the "transition operator" group of an appropriate current algebra (see Section 3 ).

If we consider hadrons in their rest systems - which is permissible if we may limit ourselves to a consideration of the compact rotation sub-groups $R_{3}^{(+)}$and $R_{3}^{(-)}$of the non-compact groups $\mathscr{P}^{(+)}$and $\mathscr{P}^{(-)}$respectively. If we next set $N_{3}=R_{3}^{(+)} \otimes R_{3}^{(-)}$, it follows that we are restricting ourselves to the compact group $\bar{W}_{3} \otimes N_{3}$ with 24 generators. Let us denote an irreducible representation of $\bar{W}_{3} \otimes N_{3}$ by $\left(R_{1}, d_{1} ; R_{2}, d_{2}\right)$ where $R_{1}\left(R_{2}\right)$ is the dimension of the irreducible representation of the $U_{3}^{(+)}\left(U_{3}^{(-)}\right)$group while $d_{1}\left(d_{2}\right)$ denotes the dimension of $R_{3}^{(+)}\left(R_{3}^{(-)}\right)$. The quantities $d_{1}$ and $d_{2}$ are, of course, related to $j_{1}$ and $j_{2}$, the angular momenta respectively of the positive and negative chiral field combinations:

$$
d_{1}=2 j_{1}+1 ; \quad d_{2}=2 j_{2}+1
$$

As before [5], the parity operation $P$ interchanges the role of $\psi_{i}^{(+)}$and $\psi_{i}^{(-)}$, i.e. $P$ demands: 


$$
P: \quad\left(R_{1}, d_{1} ; R_{2}, d_{2}\right) \longleftrightarrow\left(R_{2}, d_{2} ; R_{1}, d_{1}\right)
$$

Similarly, the charge conjugation operation $C$ requires:

$$
C: \quad\left(R_{1}, d_{1} ; R_{2}, d_{2}\right) \longleftrightarrow\left(R_{2}^{*}, d_{2} ; R_{1}^{*}, d_{1}\right)
$$

where $R_{1}^{*}\left(R_{2}^{*}\right)$ represents the conjugate representation of $R_{1}\left(R_{2}\right)$. Hence, in general, particles of opposite $P$ and $C$ [20] arise in the same irreducible representation of $\bar{W}_{3} \otimes N_{3}$. Since these questions are handled in the same way as in the $\bar{W}_{3}$ theory discussed elsewhere [5], we shall not go into detail. Instead, we focus our attention on the new features introduced into the theory of hadrons by enlarging the group to $\bar{W}_{3} \otimes N_{3}$.

When one reduces an irreducible representation of $\bar{W}_{3} \otimes N_{3}$ into those of its subgroup $\bar{W}_{3} \otimes N_{3}$, one can determine the spin values of the particles contained in the representation. The reduction of $N_{3}=R_{3}^{(+)} \otimes R_{3}^{(-)}$into the common $R_{3}$ subgroup is well-known, and a representation of $\left(R_{1}, d_{1} ; R_{2}, d_{2}\right)$ contains particles with spins $J$ given by [21]:

$$
J=\left(j_{1}+j_{2}\right),\left(j_{1}+j_{2}-1\right), \ldots\left|j_{1}-j_{2}\right|
$$

Thus, for each spin value of $J$ given in equation (9), we have an irreducible representation $\left(R_{1}, R_{2}\right)$ of $\bar{W}_{3}$. As an example, consider the case of mesons assigned to the particle representation (P.R.):

$$
\left(3,2 ; 3^{*}, 2\right) \oplus\left(3^{*}, 2 ; 3,2\right)
$$

The second representation in (10) is needed in order to achieve an eigenstate of the parity (equation 7) and hence we are forced into a parity doublet P.R. However, this P.R. is selfconjugate for the charge conjugation operation (equation 8 ) so that in this case we are not involved in charge conjugation doubling. Equation (9) then tells us that this P.R. contains four nonets with $J=0^{ \pm}$and $1^{ \pm}$. The parity doublet structure is essentially due to the $\bar{W}_{3}$ subgroup, whereas the $N_{3}$ subgroup has led to the grouping together [22] of $J=0$ and $J=1$ mesons into a larger P.R. If one wishes to group together $J=0,1$ and 2 mesons in a single P.R. of $W_{3} \otimes N_{3}$, one may choose:

$$
\left(3,3 ; 3^{*} ; 3\right) \oplus\left(3^{*}, 3 ; 3,3\right)
$$

The P.R. (11) is again self-conjugate for $C$ and leads to six nonets of $J=0^{ \pm}, 1^{ \pm}$and $2^{ \pm}$mesons; it can easily be shown that $C=+1$ for $J=0^{ \pm}, 2^{ \pm}$and $C=-1$ for $J=1^{ \pm}$. On the other hand, if one wishes to place only $J=1$ and 2 in the same P.R., one is compelled to consider:

$$
\left(3,2 ; 3^{*}, 4\right) \oplus\left(3^{*}, 4 ; 3,2\right)
$$

The P.R. (12) now raises some interesting complications. At the level of $\bar{W}_{3} \otimes R_{3}$, the $J=1$ and $J=2$ mesons are decoupled and we can assign a definite charge conjugation parity to the opposite space parity particles; we have $P$ but no $C$ doubling. However, we must now acknowledge that because of (8), the P.R. (12) is not an eigenstate of $C$ and we must take a linear combination of it with the P.R.:

$$
\left(3,4 ; 3^{*}, 2\right) \oplus\left(3^{*}, 2 ; 3,4\right)
$$


But the combination of (12) and (13) leads to both parity and charge conjugation doublets, i.e. four nonets each of $J=1$ and of $J=2$ mesons. Our preference will be for the P.R. (11) (see below).

Having explained how $\bar{W}_{3} \bigotimes N_{3}$ performs as a "spectrum-generating" group, we now show how we may use the $(V-A)$ theory to deduce mass relations between hadrons of different $J$ and $P$ (and $C$ if necessary) when we switch on the $\mathscr{L}_{m}$ and $\mathscr{L}^{\prime}$ symmetry-breaking terms; $\mathscr{L}_{m}$ is the mass part of the Lagrangian (5) and $\mathscr{L}^{\prime}$ is the medium-strong (MS) symmetry-breaking interaction defined in $I$ and specified below. Since the effect of $\mathscr{L}_{m}$ is expected to be rather large, we should consider the effect of

$$
\sum_{n=1}^{\infty}\left(\mathscr{L}_{n}\right)^{n}
$$

where $\left(\mathscr{L}_{m}\right)^{n}$ means symbolically the effective interaction in $n$-th order perturbation with respect to $\mathscr{L}_{m ;}$ we shall continue to write $\mathscr{L}_{m}$ as the symmetry-breaking term which reduced $\bar{W}_{3} \otimes N_{3}$ to $U_{3} \otimes R_{3}$ but shall understand that in an actual calculation (14) will be used. In any case, the mass splitting due to $\mathscr{L}_{m}$ (or (14)) is according to sub-multiplets belonging to different $U_{3} \otimes R_{3}$ representations until we introduce the $U_{3}$-violating interaction $\mathscr{L}^{\prime}$. At the $\bar{W}_{3}$ level, we considered two possibilities $[5]$ for the $\left(R_{1}, R_{2}\right)$ tensor structure (T.S.) of $\mathscr{L}^{\prime}$, namely:

$$
\begin{aligned}
& \mathscr{L} \sim T_{3}^{3} \cdot+T_{3}^{3^{\circ}} \\
& \mathscr{L} \sim T_{3}^{3}+T_{3}^{3^{\circ}}
\end{aligned}
$$

where the undashed (dashed) index refers to the $U_{3}^{(+)}\left[U_{3}^{(-)}\right]$group. In $I$, we were not interested in the transformation property of $\mathscr{L}^{\prime}$ with respect to the $N_{3}$ group but now we wish to specify it and we do so by resorting to the quark model. In view of the evidence presented in $I$, it is suggestive to choose:

$$
\begin{aligned}
\mathscr{L}^{\circ}= & a^{\cdot}\left\{\left(\bar{\psi}_{3} \gamma_{\mu} \Psi_{3}\right)\left(\sum_{i=1}^{3} \bar{\Psi}_{i} \gamma_{\mu} \Psi_{i}\right)+\left(\bar{\psi}_{3} \gamma_{\mu} \gamma_{5} \Psi_{3}\right)\left(\sum_{i=1}^{3} \bar{\psi}_{i} \gamma_{\mu} \gamma_{5} \Psi_{i}\right)\right. \\
& \left.-\frac{1}{3}\left[\left(\sum_{i=1}^{3} \bar{\Psi}_{i} \gamma_{\mu} \Psi_{i}\right)^{2}+\left(\sum_{i=1}^{3} \bar{\psi}_{i} \gamma_{\mu} \gamma_{5} \Psi_{i}\right)^{2}\right]\right\}
\end{aligned}
$$

Note that $\mathscr{L}^{\prime}$ is again of the form of a $(V-A)$ interaction and we assume, of course, that $\left|m^{\prime}\right| \ll|m|^{-2}$. This choice corresponds to equation (15b).

Using the chiral decomposition of $\psi_{i}$, one finds that $\mathscr{L}^{\prime}$ defined by (16) possesses the T.S. with respect to $\bar{W}_{3} \otimes N_{3}$ : 


$$
(8,1 ; 1,1) \oplus(1,1 ; 8,1)
$$

Moreover, we note that:

$$
\left[\mathbf{J}_{1}, \int d^{3} x \mathscr{L}^{\prime}(x)\right]=\left[\mathbf{J}_{2}, \int d^{3} x \mathscr{L}^{\prime}(x)\right]=0
$$

where $J_{1}$ and $J_{2}$ are the angular momentum operators with respect to $R_{3}^{(+)}$and $R_{3}^{(-)}$respectively. Equation (18) implies that the interaction Hamiltonian:

$$
H^{\cdot}=-\int d^{3} x \mathscr{L}^{\prime}(x)
$$

is a scalar with respect to $N_{3}$. We may therefore compute the mass splitting of particles in our theory by taking the expectation values of the operator $\left(H_{m}=\int d^{3} x \mathscr{L}_{m}\right)$ :

$$
\Delta E=H_{m}+H^{\prime}
$$

The effect of $H_{m}$ is simply to remove the mass degeneracy with respect to different spin, parity and $U_{3}$ sub-groups whereas $H^{\prime}$ removes the mass degeneracy within $U_{3}$.

We now wish to demonstrate that the general mass formula, say for mesons, under the action of (20) will have the form:

$$
m^{2}=m_{0}(\alpha, J, P)+m_{1}(\alpha, \beta)
$$

where $m_{0}$ is the contribution from $H_{m}$ and depends on spin $J$, parity $P$ and the chief $U_{3}$ quantum number $\alpha$ but is independent of the $U_{3}$ sub-quantum numbers $\beta$ (i.e. $I$ and $Y$ ). On the other hand, $m_{1}$ is the contribution from $H^{\prime}$ and depends upon $\alpha$ and $\beta$, but is common for the same $U_{3}$ representation with different $J$ and $P$. The nature of $m_{0}$ follows directly from the fact that $H_{m}$ mixes $R_{3}^{(+)}$and $R_{3}^{(-)}$in addition to $U_{3}^{(+)}$and $U_{3}^{(-)}$leading to a reduction of the initial $\bar{W}_{3} \otimes N_{3}$ group to $U_{3} \otimes R_{3}$. It requires more care to prove the assertions concerning $m_{1}$. Consider first the mass degeneracy with respect to the spin value $J$; a particle with $\operatorname{spin} J$ and $z$-component $M$ can be decomposed by means of (9):

$$
\left|J, M ; \omega>=\sum_{m_{1}, m_{2}} C\left(J, j_{1}, j_{2} ; M, m_{1}, m_{2}\right)\right| j_{1}, m_{1} ; j_{2}, m_{2} ; \omega>
$$

where $C\left(J, j_{1}, j_{2} ; M_{1}, m_{1}, m_{2}\right)$ is the Clebsch-Gordon coefficient for the coupling of two spins $j_{1}$ and $j_{2}$ into $J$ and $\omega$ refers to all other quantum numbers such as parity, $\bar{W}_{3}$ indices, etc. The next step is to argue that because of the invariance of $H^{\prime}$ with respect to $N_{3}$ (equation 18), we may write:

$$
\begin{aligned}
& <j_{1}^{\prime}, m_{1}^{\prime} ; j_{2}^{\prime}, m_{2}^{\prime} ; \omega\left|H^{\circ}\right| j_{1}, m_{1} ; j_{2}, m_{2} ; \omega> \\
& =\delta_{j_{1}^{\prime} j_{1}} \delta_{j_{2}^{\prime} j_{2}} \delta_{m_{1}^{\prime} m_{1}} \delta_{m_{2} m_{2}}<j_{1}, j_{2} ; \omega^{\circ}\left\|H^{\circ}\right\| j_{1}, j_{2} ; \omega>
\end{aligned}
$$

Further, taking account of the orthogonality of the Clebsch-Gordon coefficients, equation (22) yields:

$$
\left\langle J, M ; \omega^{\circ}\left|H^{\prime}\right| J, M, \omega\right\rangle=\left\langle j_{1}, j_{2} ; \omega^{\circ}\left\|H^{\prime}\right\| j_{1}, j_{2} ; \omega\right\rangle
$$


The important point is that the r.h.s. of equation (24) is independent of the total spin $J$ and hence $m_{1}$ (equation 21) is independent of the spin value $J$ for all possible values allowed by equation (9). Similarly, we can show that $m_{1}$ is also independent of parity in general because two parity states are given by:

$$
\begin{aligned}
& |P=+1\rangle=\frac{1}{\sqrt{2}}\left[\left|R_{1}, R_{2}\right\rangle+R_{2},\left|R_{1}\right\rangle\right] \\
& |P=-1\rangle=\frac{1}{\sqrt{2}}\left[\left|R_{1}, R_{2}\right\rangle-R_{2},\left|R_{1}\right\rangle\right]
\end{aligned}
$$

where, for simplicity, we have neglected the spin indices and omitted a possible complication due to charge conjugation doublets. Then, we must compute:

$$
\begin{aligned}
& \Delta E_{+}=\left\langle P=+1\left|H^{\prime}\right| P=+1\right\rangle \\
& \Delta E_{-}=\left\langle P=-1\left|H^{\circ}\right| P=-1\right\rangle
\end{aligned}
$$

Both mass displacements in (26) would be equal if:

$$
<R_{1}, R_{2}\left|H^{\prime}\right| R_{2}, R_{1}>=0
$$

Equation (27) is certainly satisfied for the choice of $\mathscr{L}^{\prime}$ given by equation (16) except when $R_{1}=R_{2}$. In this argument, we have neglected complications due to charge conjugation doublets since then equation (25) is insufficient to define particles with definite charge conjugation parity. In that case, the representations of particles with $C= \pm 1$ would be in general given by:

$$
\begin{aligned}
\mid P=+1, C= \pm 1>= & \frac{1}{2}\left\{\left|R_{1}, j_{1} ; R_{2}, j_{2}>+\right| R_{2}, j_{2} ; R_{1}, j_{1}\right. \\
& \left.\left. \pm\left|R_{2}^{*}, j_{2} ; R_{1}^{*}, j_{1}> \pm\right| R_{1}^{*}, j_{1} ; R_{2}^{*}, j_{2}\right\rangle\right\}
\end{aligned}
$$

where now we have explicitly referred to the spins $j_{1}$ and $j_{2}$. With the P.R.'s (28), the independence of the mass splitting due to $H^{\prime}$ with respect to $P$ and $C$ is still, in general, maintained unless we have $R_{1}=R_{2}^{*}$ and $j_{1}=j_{2}$. Apart from this exception, $m_{1}$ depends only upon its $\bar{W}_{3}$ sub-quantum numbers $\alpha$ and $\beta$ which we have already investigated in $I$. Thus, we have proved the validity of the mass formula (21) for mesons under assumptions which follow plausibly from the $(V-A)$ quark model with chiral decomposition.

Actually, the tensor structure of the symmetry-breaking term $\mathscr{L}^{\prime}$ given by equation (16) is supported by the mass spectra and decays of the $J=1$ and $J=2$ mesons (cf. I and reference $[22])$. Indeed, it is tempting to place all the known $J=0,1$ and 2 mesons in the same particle representation of the $\bar{W}_{3} \otimes N_{3}$ group; this leads quite naturally to the P.R. (11) and to the use of the T.S. (17) for $\mathscr{L}^{\prime}$. We may therefore take over the mass formula (21) where the structure of $m_{1}(\alpha, \beta)$ is determined by our knowledge of the P.R. $\left[\left(3,3^{*}\right) \pm\left(3^{*}, 3\right)\right]$ and the T.S. 
of $\mathscr{L}^{\prime}[(8,1)+(1,8)]$ within the framework of the $\bar{W}_{3}$ group. This was investigated previously in $I$ where we derived the result:

$$
K\left(J^{+}\right)-\pi\left(J^{+}\right)=K\left(J^{-}\right)-\pi\left(J^{-}\right)
$$

where $K\left(J^{P}\right)$ and $\pi\left(J^{P}\right)$ denote the $m^{2}$ of $K$-type and $\pi$-type mesons respectively with spin $J$ and parity $P$. The implication of equation (21) is that equation (29) now holds for the nonets of mesons with $J=0,1$ and 2 as well as with opposite parity, namely:

$$
\begin{aligned}
& K\left(2^{+}\right)-\pi\left(2^{+}\right)=K\left(1^{+}\right)-\pi\left(1^{+}\right)=K\left(0^{+}\right)-\pi\left(0^{+}\right) \\
& =K\left(2^{-}\right)-\pi\left(2^{-}\right)=K\left(1^{-}\right)-\pi\left(1^{-}\right)=K\left(0^{-}\right)-\pi\left(0^{-}\right)
\end{aligned}
$$

In addition, of course, the "Schwinger" formula [23] must hold separately for each of the six nonets $J=0^{ \pm}, 1^{ \pm}, 2^{ \pm}$. If we insert numbers, equation (30) as well as the individual "Schwinger" formulas are fairly well satisfied [24] but since much of the experimental information is still preliminary, we do not wish to press the agreement with theory. However, it should be remarked that equation (30) contains as one of its predictions a major argument for assigning the $J=0^{-}$ and $J=1^{-}$mesons to the " 35 " representation of $S U_{6}$, namely that:

$$
K\left(1^{-}\right)-\pi\left(1^{-}\right)=K\left(0^{-}\right)-\pi\left(0^{-}\right)
$$

If equation (30) holds up for the $J=2$ mesons as well as the opposite parity mesons, the particle representation (11) would acquire great interest [25]. In terms of our quark model, this P.R. requires us to couple two quark-antiquark pairs with no relative orbital angular momentum. The unit spin for each quark-antiquark pair follows from the requirement that only a left-handed quark and a right-handed antiquark can be combined together. Our six nonets of $J=0^{ \pm}, 1^{ \pm}$and $2^{ \pm}$would, on this picture, be contructed out of two quark-antiquark pairs which is certainly not unreasonable.

Thus far, we have shown how to construct a theory of mesons on the basis of the $W_{3} \otimes N_{3}$ group, regarded as a "spectrum-generating" group. A theory of baryons can also be constructed on the basis of this larger group with due regard paid to the usual complication of building up 4 -component spinors for the hadrons out of the 2-component quark fields $\psi_{1}^{(+)}$and $\psi_{1}^{(-)}$(in order to define the parity). The procedure is essentially the same as in the $\bar{W}_{3}$ case $[1]$ and we need not go into detail. Suffice to say, we may assign the P.R.:

$$
(3,2 ; 6,3) \oplus\left(6^{*}, 3 ; 3,2\right)
$$

to the lowest mass baryons and we would then expect to have $S U_{3}$ octet and decuplet baryons with $J=1 / 2$ and $J=3 / 2$. The relative parity of these particles would be determined by whether the effect of the symmetry-breaker $\mathscr{L}_{m}$ must be computed to first order or to higher orders. The P.R. (32) could easily reduce at the $U_{3} \otimes R_{3}$ level to [26]:

$$
\begin{array}{ll}
J=1 / 2^{+} \text {octet; } & J=3 / 2^{+} \text {decuplet } ; \\
J=1 / 2^{-} \text {decuplet } ; & J=3 / 2^{-} \text {octet }
\end{array}
$$

Adding the symmetry-breaking $\mathscr{L}^{\prime}$ with a tensor structure (17) would give rise to a mass formula analogous to (21) but we shall not write this down. We merely note that the P.R. (32) is rather attractive for the lowest mass baryons if the P.R. (11) is appropriate for the lowest mass 
mesons; a di-quark with unit spin is combined with a $J=1 / 2$ quark triplet.

\section{3. $\bar{W}_{3} \otimes N_{3}$ Group as a Current Algebra}

Rather than pursue further the consequences of the $\bar{W}_{3} \otimes N_{3}$ group as a "spectrum-generating" group, we wish to point out the possibility of regarding the 24 generators of this group as defining a current algebra which goes beyond the current algebra defined by the 18 generators of $\bar{W}_{3}$ group. We recall that the generators of the $U_{3}^{(+)}$and $U_{3}^{(-)}$groups defined by :

$$
\left.A^{(t)}\right) j_{i}^{j}(t)=-\frac{1}{2} \int d^{3} x \bar{\psi}_{i} \gamma_{4}\left(1 \pm \gamma_{5}\right) \Psi_{i}
$$

obey the equal-time commutation relations:

$$
\begin{aligned}
& \left.\left.\left.\left.\left[A^{(+)}\right)_{i, A^{(+)}}^{j}\right)_{K}^{l}\right]=\delta_{K}^{j} A^{(+)}\right)_{i}^{l}-\delta_{i}^{l} A^{(+)}\right)_{K}^{j} \\
& \left.\left.\left.\left.\left[A^{(-)}\right){ }_{i}^{j}, A^{(-)}\right){ }_{K}^{l}\right]=\delta_{K}^{j} A^{(-)}\right){ }_{i}^{l}-\delta_{i}^{l} A^{(-)}\right){ }_{K}^{j} \\
& \left.\left.\left[A^{(+)}\right)_{i, A^{(-)}}^{j}\right)_{K}^{l}\right]=0
\end{aligned}
$$

If we define positive and negative linear combinations of $\left.A^{(+)}\right)_{i}^{j}$ and $\left.A^{(-)}\right)_{i}^{j}$, to wit:

$$
\begin{aligned}
& \left.A_{i}^{j}=\left[A^{(+)}\right){ }_{i}^{j}+A^{(-)}\right){ }_{i}^{j} \\
& \left.\left.B_{i}^{j}=\left[A^{(+)}\right){ }_{i}^{j}-A^{(-)}\right) \begin{array}{l}
j \\
i
\end{array}\right]
\end{aligned}
$$

then the new quantities must satisfy the commutation relations:

$$
\begin{aligned}
& {\left[\begin{array}{ll}
A_{i}^{j}, & A_{K}^{l}
\end{array}\right]=\delta_{K}^{j} A_{i}^{l}-\delta_{i}^{l} A_{K}^{j}} \\
& {\left[\begin{array}{ll}
B_{i}^{j}, & B_{K}^{l}
\end{array}\right]=\delta_{K}^{j} A_{i}^{l}-\delta_{i}^{l} A_{K}^{j}} \\
& {\left[A_{i}^{j}, B_{K}^{l}\right]=\delta_{K}^{j} B_{i}^{l}-\delta_{i}^{l} B_{K}^{j}}
\end{aligned}
$$

From the definitions (34), it follows that: 


$$
\begin{aligned}
& A_{i}^{j}(t)=-\int d^{3} x \bar{\psi}_{j} \gamma_{4} \psi_{i} \\
& B_{i}^{j}(t)=-\int d^{3} x \bar{\psi}_{j} \gamma_{4} \gamma_{5} \psi_{i}
\end{aligned}
$$

The generators (38a) and (38b) are the integrals over all space of the fourth components of the vector and axial vector quark currents respectively. We may think of the quark currents as replaced by the physical hadron currents and continue to use the equal-time commutation relations defined by (37). This was Gell-Mann's starting point and explains the connection with the $\bar{W}_{3}$ group of the $(V, A)$ quark model with chiral decomposition.

The current algebra suggested by the maximal chiral group $\bar{W}_{3} \otimes N_{3}$ of the quark model can be derived following the above procedure. If we recall that we are dealing only with particles at rest, we may neglect the orbital angular momentum of the left and right-handed quark systems and consider only the spin contributions; the generators of $R_{3}^{( \pm)}$are then defined by:

$$
J_{\mu \nu}^{(t)}(t)=\frac{1}{2} \sum_{i=1}^{3} \int d^{3} x \psi_{i}^{*}\left[\gamma_{\mu}, \gamma_{\nu}\right]\left(\begin{array}{lll}
1 \pm \gamma_{5}
\end{array}\right) \psi_{i}
$$

It is to be noted that in the definition (39), a trace is being taken in each $U_{3}$ space. We now show that the generators $J_{\mu \nu}^{(+)}, J_{\mu \nu}^{(-)}$can be expressed as linear combinations of a certain subgroup of generators of the chiral $W_{6}$ group [12]; thus, if we define

$$
\begin{aligned}
& \bar{V}_{\mu}=\sum_{i=1}^{3} \int d^{3} x \bar{\psi}_{i} \gamma_{\mu} \psi_{i} \\
& \bar{A}_{\mu}=\sum_{i=1}^{3} \int d^{3} x \bar{\psi}_{i} \gamma_{\mu} \gamma_{5} \psi_{i}
\end{aligned}
$$

then we have:

$$
\begin{aligned}
& J_{\mu \nu}^{(+)}+J_{\mu \nu}^{(-)}=-\varepsilon_{4 \mu \nu \lambda} \bar{A}_{\lambda}+\delta_{\mu 4} \bar{V}_{\nu}-\delta_{\nu 4} \bar{V}_{\mu} \\
& J_{\mu \nu}^{(+)}-J_{\mu \nu}^{(-)}=-\varepsilon_{4 \mu \nu \lambda} \bar{V} \lambda+\delta_{\mu 4} \bar{A}_{\nu}-\delta_{\nu 4} \bar{A}_{\mu}
\end{aligned}
$$

In equations (41a) and (41b), two of the generators $\bar{V}_{4}$ and $\bar{A}_{4}$, are already contained among the generators of $\bar{W}_{3}$ since $\bar{V}_{4} \equiv-\sum_{i=1}^{3} A_{i}^{i}$ and $\bar{A}_{4} \equiv-\sum_{i=1}^{3} B_{i}^{i}$ (cf. equations 38a and 38b). The totality 
of 24 generators - the 18 of $\bar{W}_{3}$ and the 6 of $N_{3}$ - form a closed system and the current algebra associated with these generators - reinterpreted in terms of physical hadron currents - is the proposed generalization of Gell-Mann's original current algebra. Some of the consequences of this current algebra between the physical hadron states, following the procedure of Fubini and Furlan [27] will be discussed elsewhere.

It is apparent from the above that the 24 generators of the $\bar{W}_{3} \otimes N_{3}$ group constitute a subgroup of the 72 generators of the chiral $W_{6}$ group. This explains why some of the consequences of the $\bar{W}_{3} \otimes N_{3}$ group - treated as a "spectrum-generating" group - bear a resemblance to some of the results obtained with the chiral $W_{6}$ theory. However, it seems that the chiral $W_{6}$ group is too large to be useful either as a "spectrum-generating" or as a "transition operator" group. It is conceivable that the maximal chiral group, $\bar{W}_{3} \otimes N_{3}$, suggested by the quark model may serve this double purpose or at least prove useful to motivate a current algebra which beyond the one used so successfully at present. Certainly, if experiment supports this large chiral group as both a "spectrum-generating" and "transition operator" group, it would then be justified to look into the deeper meaning of the $(V-A)$ quark model with chiral decomposition [15].

\section{References}

1. The chiral groups are discussed in R.E. MARSHAK and S. OKUBO, Nuovo Cim. 19, 1226 (1961) (especially Appendix); see also R. MARSHAK, N. MUKUNDA and S. OKUBO, Phys. Rev. 137, B698 (1965) and MARSHAK, OKUBO, SCHECHTER and WOJTASZEK, Supplement to Prog. Theor. Phys. (1965). The first paper dealt with an arbitrary number $n$ of Dirac fields interacting via a four-fermion interaction; when $n=3$, the fields must carry fractional charge, hypercharge and baryon number and become "quarks" (cf. M. GELL-MANN, Phys. Let ters 8, 214, 1964).

2. The non-chiral groups are discussed in S. OKUBO and R.E. MARSHAK, Phys. Rev. Lett. 13, 818 (1964); ibid. 14, 156 (1965); we called these groups "Casimir" groups but we accept the more recent term "non-chiral". See also; K. BARDAKCI et al., ibid. 14, 48 (1965).

3. The differences between the chiral and non-chiral groups are discussed in $S$. OKUBO and R.E. MARSHAK, Proceedings of the Coral Gables Conference on Symmetries (1965); see also R.E. MARSHAK, Proceedings of the International Conference on Elementary Particles, Kyoto (1965).

4. We shall use the symbol $\mathscr{D}$ for the group representing the discrete transformations $P, C$. $T$.

5. R. MARSHAK, S. OKUBO and J. WOJTASZEK, Phys. Rev. Let ters 15, 463 (1965); this paper will hereafter be referred to as $I$.

6. M. GELL-MANN, Phys. Rev. 125, 1067 (1962); see also M. GELL-MANN, Physics 1, 63 (1964).

7. The difference between $S \bar{W}_{3}$ and $\bar{W}_{3}$ arises from the extra gauge degree of freedom in the quark model [cf. I].

8. Y. NE' EMAN, Lectures at the Pacific Summer School, Honolulu (1965); we do not distinguish between "spectrum-generating" and "approximate symmetry" groups since we limit ourselves to compact groups.

9. It is possible to develop a quark model which gives rise to a compact group as large as $U(12)$ but one is then in trouble with the baryon number (cf. second paper of reference $[2])$. The relation between the non-chiral compact group $W_{6}$ and the non-compact $\tilde{U}_{(12)}$ was first discussed by BARDAKCI, CORNWALL, FREUND and LEE (Phys. Rev. Let ters 14, 48, 1965); see the more complete table in the Appendix to the present paper. 
10. Cf. R.F. DASHEN and M. GELL-MANN, Phys. Let ters 17, 142 and 145 (1965).

11. The collinear subgroup $S U_{W}(6)$ of the non-chiral $W_{6}$ has been discussed most fully by J.J. LIPKIN and S. MESHKOV (cf. Phys. Rev. Let ters 14, 670, 1965); recently, J.D. JACKSON (Phys. Rev. Letters 15, 990, 1965) has shown that this group is contradicted by many experiments.

12. R. FEYNMAN, M. GELL-MANN and R. ZWEIG, Phys. Rev. Letters 13, 678 (1964).

13. Cf. W. ALLES and D. AMATI, CERN preprint (1965).

14. Another way of saying this is that somehow the mass term can be subtracted off when one focuses on the physical currents.

15. The $(V-A)$ four-fermion interaction considered in the quark model (where the parity is conserved) should not be confused with the parity-violating $(V-A)$ weak interaction; for a possible connection between the $(V-A)$ strong interaction quark model for hadrons and the universal ( $V-A)$ weak interaction theory for hadrons and leptons, see the second paper of reference $[3]$.

16. The group $\mathscr{B}$ is reminiscent of Yukawa's theory which postulates two independent "inner" and "outer" Poincaré spaces; (cf. Prog. Theor. Phys. 31, 1167, 1964); however, in contrast to Yukawa, we have been led to two spaces within the framework of an original single Poincaré space and we do not employ one of the spaces to derive the internal symmetries.

17. By switching on the difference between the $V$ and $A$ coupling constants, namely ( $\left.g_{V}-g_{A}\right)$, we could pass through the subgroup $\bar{W}_{3} \bigotimes \mathscr{P}$; we prefer to omit this step by switching on $\mathscr{L}_{m}$ so that we proceed directly from $\bar{W}_{3} \otimes \mathscr{B}$ to $U_{3} \otimes \mathscr{P}$.

18. S. COLEMAN, private communication; we thank Prof. Coleman for a useful discussion of this point.

19. S. COLEMAN, Phys. Rev. 138, B1262 (1965).

20. In principle, the problem of charge conjugation doubling confronted us in the simple $\bar{W}_{3}$ theory but it was not necessary to deal with this question for the lowest particle representations actually considered.

21. Strictly speaking, $j_{1}$ and $j_{2}$ combine to give the range $J=0,1,2 \ldots$ when we include the relative orbital angular momentum $L$; if we restrict ourselves to $L=0$, equation (9) follows:

22. In I where only the mass spectra were considered on the basis of the $\bar{W}_{3}$ theory, equal credibility was given to the P.R. assignment $[(8,1) \pm(1,8)]$ and $\left[\left(3,3^{*}\right) \pm\left(3^{*}, 3\right)\right]$ for the $J=0$ mesons whereas the latter P.R. was definitely favored for the $J=1$ and $J=2$ mesons. Recent calculations by $W$. Moran (to be published) on the decay of $J=1$ and $J=2$ mesons into $J=0$ mesons also tends to favor the latter assignment for the $J=0$ mesons. This allows us to group together the $J=0,1,2$ mesons in the same P.R. of $\bar{W}_{3} \bigotimes N_{3}$ (see below).

23. The "Schwinger" formula was derived originally for vector mesons on the basis of a specific model (J. SCHWINGER, Phys. Rev. 135, B816, (1964); we showed in I that this formula follows automatically for any $J^{P}$ nonet in the $\bar{W}_{3}$ theory for a P.R. $\left[\left(3,3^{*}\right),\left(3^{*}, 3\right)\right]$ and a T.S. $[(8,1)+(1,8)]$.

24. We have shown in I that the "Schwinger" formula holds well for the $J=1^{-}$and $J=2^{+}$nonets; the discrepancy for the $J=0^{-}$nonet (wherein $X$ is considered to be the ninth pseudoscalar 
meson) may be due to the fact that $X$ belongs to an $I=1$ triplet and has nothing to do with the usual $J=0^{-}$octet [see W. KIENZLE et al., Phys. Letters 19, 438, 1966]. Equation (30) is reasonably well satisfied if we follow I in assigning $J=2-$ to the $A_{1}$ meson. It must be admitted that the situation has been confused by recent evidence favoring $J=2^{-}$for the $A_{2}$ meson and the possible non-existence of $A_{1} !$ [see F. LEFEBVRES et al. Phys. Letters 19, 434, 1966].

25. It should be pointed that while our theory assigns $J=0^{--}, 0^{+-}, 1^{-+}, 1^{++}, 2^{--}, 2^{+-}$to the lowest mass $\pi$-type mesons, the $S U_{6}$ theory (and the non-relativistic quark model) agrees with five of these assignments but differs in preferring $J=1^{+-}$to $J=2^{--}$. This critical difference as well as an alternative set of mass relations to (30) (cf. R. GATTO, L. MAIANI and G. PREPARATA, Phys. Rev. 14, 70, 1965) should permit a decision between the two models.

26. The $J=1 / 2^{-}$decuplet plus $J=3 / 2^{-}$octet would comprise a "52-" $P$. R. in our theory compared to the "70-n P.R. in the $S U_{6}$ theory for which some arguments have been given (cf. I.P. GUYK and S.P. TUAN, Phys. Rev. 140, B164, 1965); the experimental data are not sufficient to decide between these two alternatives at the present time.

27. S. FUBINI and G. FURLAN, Physics, 1, 229 (1965) and subsequent papers. Recently, the $S U_{3}$ current algebra (S. FUBINI, G. FURLAN and D. ROSSETTI, Trieste Report, 1965) has been used to derive the $S U(3)$ mass formula; this opens up the possibility of using the $\bar{W}_{3} \bigotimes N_{3}$ current algebra to derive mass relations without any further guidance from the quark model.

\section{Appendix}

It is important, in order to appreciate the unique properties of the group $\bar{W}_{3} \otimes B$ considered in the text, to determine all the groups admissible with the quark model. Explicitly, we are looking for all groups which contain $U_{3} \otimes \mathscr{P}$ as a subgroup, and conserve parts of the Lagrangian $\mathscr{L}_{K}+\mathscr{L}_{m}+\mathscr{L}_{I}$ [cf. equation (1) in the text]. In order to simplify the discussion, we shall restrict ourselves to the proper orthochronous Lorentz group $L_{+}^{\uparrow}$ rather than the Poincare group $\mathscr{P}$. Furthermore, we assume throughout that a definite baryon number can be assigned to the triplet of quarks (or if one prefers the 12-component quark $\psi$ - see below).

Since the quark Lagrangian is a polynomial (of the 4-th degree) in the 12-dimensional $\psi$ and $\psi^{+}$, it is clear that the largest relevant group must be some "quasi-unitary" group, namely, a group $\tilde{U}_{B}(12)=\{A\}$, the elements $A$ of which fulfill $A^{+} B A=B$, or $A^{+} B=B A^{-1}$ for a fixed $12 \times 12$ matrix $B$; here $B$ must not be the identity matrix (although $B$ is Hermitian). Since $\tilde{U}_{B}(12) \supset L_{+}^{\uparrow} \otimes S U_{3}, B$ must be a combination of $\gamma_{0} \otimes I_{3}$ and $i \gamma_{5} \gamma_{0} \otimes I_{3}$ (where $I_{3}$ is the 3dimensional identity matrix):

$$
B=\left(\alpha+\beta_{i} \gamma_{5}\right) \gamma_{0} \otimes I_{3} ; \quad \alpha, \beta \text { real; } \alpha^{2}+\beta^{2}=1 ; B^{2}=I
$$

When some particular $B$ is chosen, define

$$
\bar{\psi}=\psi^{+} B
$$

It follows that when $\psi \rightarrow A \psi, \quad \bar{\psi} \rightarrow \bar{\psi} A^{-1}$.

Let $E_{k}^{i}(1 \leqslant i, k \leqslant 12)$ be a $12 \times 12$ matrix with unity at the intersection of the $i$-th row and $k$-th column and 0 everywhere else. Denote 


$$
T_{K}^{i}=\bar{\psi} E_{K}^{i} \Psi
$$

Lemma. When $\psi \rightarrow A \psi, \quad T_{K}^{i} \rightarrow\left(A^{-1}\right)_{p}^{i} A_{R}^{q} T_{q}^{p}$

Proof. The $E_{k}^{i}$ form an algebra with the rule of multiplication

$$
E_{K}^{i} E_{s}^{r}=\delta_{K}^{r} E_{s}^{i}
$$

Also, the $E_{k}^{i}$ form a linear basis for the algebra of all $12 \times 12$ matrices in the sense that each such matrix $A$ may be written in the form:

$$
A=A_{i}^{k} A_{k}^{i}
$$

$A_{k}^{i}$ are complex numbers. According to the foregoing:

$$
\begin{aligned}
T_{k}^{i} \rightarrow T_{k}^{\prime i} & =\bar{\psi} A^{-1} E_{k}^{i} A \psi=\left(A^{-1}\right){ }_{p}^{q} A_{r}^{s} \bar{\psi} E_{q}^{p} E_{k}^{i} E_{s}^{p} \psi \\
& =\left(A^{-1}\right){ }_{p}^{i} A_{k}^{s} T_{p}^{s}
\end{aligned}
$$

According to (A.6), the only bilinear invariant under $\tilde{U}(12)$ is $T_{i}^{i}$. There are, however, two invariants of the 4-th degree: $\left(T_{i}^{i}\right)^{2}$ and $T_{k}^{i}, T_{i}^{k}$. Let $S p(12)$ be a symplectic subgroup of $\tilde{U}(12)$ with some invariant tensor $G^{i k}=-G^{i k}$. If $L_{+}^{\uparrow} \otimes S U_{3}$ is a subgroup of $S p(12)$, then either

$$
G=i \sigma_{31} \otimes I_{3}, \quad \text { or } \quad i \gamma_{5} \sigma_{31} \otimes I_{3} ; \quad G^{2}=-I
$$

in a representation in which $\gamma_{5}$ is diagonal. If we use a general $\gamma$-matrix representation, then $\sigma_{31}$ can be replaced by the ordinary charge conjugation matrix $C$. The tensor $G^{i k}$ mas be used to raise and lower indices, and therefore no real distinction exists between covariant and contravariant indices. Accordingly, we define:

$$
\begin{aligned}
& \sqrt{2} T_{i k}^{(s)}=G_{i p} T_{k}^{p}+G_{k p} T_{i}^{p} \\
& \sqrt{2} T_{i k}^{(A)}=G_{i p} T_{k}^{p}-G_{k p} T_{i}^{p}
\end{aligned}
$$

$T_{i k}^{(s)} T^{(s) i k}$ and $T_{i k}^{(A)} T^{(A) i k}$ are invariant under $S p(12)$.

It is readily verified that the following chain of groups exists

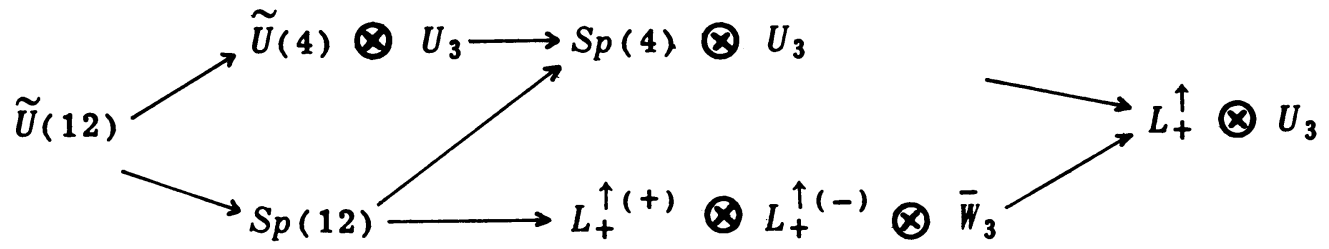


Table 1 summarizes the correspondence between groups and Lagrangians.

TABLE 1

Lagrangians and corresponding symmetry groups for $B=\gamma_{0} \otimes I_{3}$

\begin{tabular}{l|l|l}
\hline Lagrangian & Symmetry group & $\begin{array}{c}\text { Maximal compact } \\
\text { subgroup }\end{array}$ \\
\hline $\mathscr{L}_{k}$ & $L_{+}^{\uparrow(+)} \otimes L_{+}^{\uparrow(-)} \otimes \bar{W}_{3}$ & $N_{3} \otimes \bar{W}_{3}$ \\
$\mathscr{L}_{m}$ & $\tilde{U}_{(12)}$ & $\bar{W}_{6}$ \\
$S$ or $T_{k}^{i} T_{i}^{k}$ & $\tilde{U}(12)$ & $\bar{W}_{6}$ \\
$T_{i k}^{(s)} T^{(s) i k}$ or $T_{i k}^{(A)} T^{(A) i k}$ & $S p(12)$ & $U S p(6) \otimes U S p(6)$ \\
$-P+V-A+T$ & $\tilde{U}_{(4) \otimes U_{3}}$ & $N_{3}^{(d)} \otimes U_{3}$ \\
$-P+V$ or $-A+T$ & $S p_{1}(4) \otimes U_{3}$ & $N_{31} \otimes U_{3}$ \\
$-P-A$ or $V+T$ & $S p_{2}(4) \otimes U_{3}$ & $N_{31} \otimes U_{3}$ \\
$V-A$ & $L_{+}^{\uparrow(+)} \otimes L_{+}^{\uparrow(-)} \otimes \bar{W}_{3}$ & $N_{3} \otimes \bar{W}_{3}$ \\
$V$ or $A$ & $L_{+}^{\uparrow} \otimes \bar{W}_{3}$ & $S U_{2} \otimes \bar{W}_{3}$
\end{tabular}

In Table 1, $S p_{1}(4)$ leaves invariant $i \sigma_{31}$ whereas $S p_{2}(4)$ leaves invariant $i \sigma_{31} \gamma_{5} . N_{3}, N_{31}$ are isomorphic to $S U_{2} \otimes S U_{2}$, but not identical with each other. $N_{3}^{(d)}$ is isomorphic to $N_{3} \otimes\left(\begin{array}{cc}\alpha I_{2} & 0 \\ 0 & \alpha^{*} I_{2}\end{array}\right)$, where $I_{2}$ is the $2 \times 2$ identity matrix and $|\alpha|=1$. USp(6) is the unitary symplectic group in 6 dimensions. We recall that $N_{3}=R_{3}^{(+)} \otimes R_{3}^{(-)}$.

A similar table may be obtained by choosing $i \gamma_{5} \gamma_{0} \otimes I_{3}$ as the Hermitian form $B$. This will introduce $U^{(p)}(12)$ associated with the pseudoscalar four-fermion interaction; corresponding changes will be induced in some other interactions, as summarized in Table 2.

In Table 2, the superindex $(p)$ is inserted whenever the definition of the relevant object depends on the fact that $B=i \gamma_{5} \gamma_{0} \otimes I_{3}$ rather than $B=\gamma_{0} \otimes I_{3}$.

According to the above tables, two properties single out $L_{+}^{\uparrow(+)} \otimes L_{+}^{\uparrow(-)} \otimes \bar{W}_{3}$ :

(1) It is the maximal group which leaves invariant both $\mathscr{L}_{k}$ and a pure current-current interaction; $N_{3} \otimes \bar{W}_{3}$ is the maximal compact subgroup possessing the same property.

(2) It is the intersection of all groups $\tilde{U}(12)$ which contain $L_{+}^{\uparrow} \otimes U_{3}$. 
TABLE 2

Analog of Table 1 for $B=i \gamma_{5} \gamma_{0} \otimes I_{3}$

\begin{tabular}{l|l|l}
\hline Lagrangian & Symmetry group & $\begin{array}{c}\text { Maximal compact } \\
\text { subgroup }\end{array}$ \\
\hline$P$ or $T_{k}^{(p) i} T_{i}^{(p) k}$ & $\widetilde{U}^{(p)}(12)$ & $\bar{W}_{6}$ \\
$T_{i k}^{(p s)} T^{(p s) k i}$ or $T_{i k}^{(p A)} T^{(p A) i k}$ & $S^{(p)}(12)$ & $U S p(6) \otimes U S p(6)$ \\
$-S+V-A+T$ & $\tilde{U}^{(p)}(4) \otimes U_{3}$ & $N_{3}^{(p)} \otimes U_{3}$ \\
$-S+V$ or $-A+T$ & $S p_{1}^{(p)}(4) \otimes U_{3}$ & $N_{31} \otimes U_{3}$ \\
$-S-V$ or $V+T$ & $S p_{2}^{(p)}(4) \otimes U_{3}$ & $N_{31} \otimes U_{3}$
\end{tabular}

